This item was submitted to Loughborough's Research Repository by the author.

Items in Figshare are protected by copyright, with all rights reserved, unless otherwise indicated.

\title{
The considerations and limitations of feedback as a strategy for behaviour change
}

PLEASE CITE THE PUBLISHED VERSION

http://dx.doi.org/10.1080/19397038.2015.1006299

\section{PUBLISHER}

Taylor and Francis Ltd (@ 2015 the authors)

\section{VERSION}

VoR (Version of Record)

\section{PUBLISHER STATEMENT}

This work is made available according to the conditions of the Creative Commons Attribution 4.0 International (CC BY 4.0) licence. Full details of this licence are available at: http://creativecommons.org/licenses/ by/4.0/

\section{LICENCE}

CC BY-NC-ND 4.0

\section{REPOSITORY RECORD}

Wilson, Garrath T., T.A. Bhamra, and Debra Lilley. 2019. "The Considerations and Limitations of Feedback as a Strategy for Behaviour Change”. figshare. https://hdl.handle.net/2134/17195. 
This article was downloaded by: [Loughborough University]

On: 17 March 2015, At: 02:58

Publisher: Taylor \& Francis

Informa Ltd Registered in England and Wales Registered Number: 1072954 Registered office: Mortimer House, 37-41 Mortimer Street, London W1T 3J H, UK

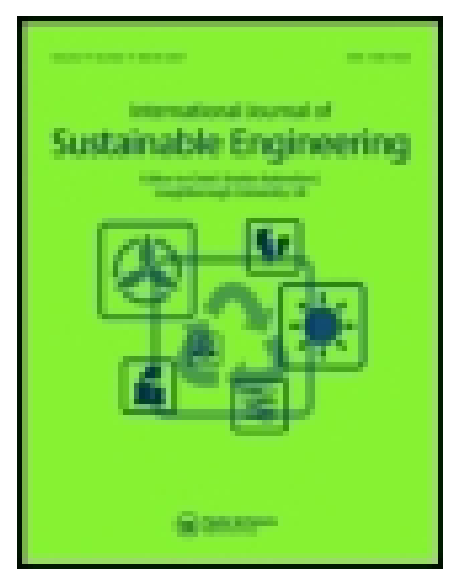

\section{International J ournal of Sustainable Engineering}

Publication details, including instructions for authors and subscription information: http:// www.tandfonline.com/loi/ tsue20

\section{The considerations and limitations of feedback as a strategy for behaviour change}

\author{
Garrath T. Wilson ${ }^{a}$, Tracy Bhamra ${ }^{a} \&$ Debra Lilley ${ }^{a}$ \\ a Loughborough Design School, Loughborough, UK \\ Published online: 12 Mar 2015.
}

\section{(DrossMark}

Click for updates
To cite this article: Garrath T. Wilson, Tracy Bhamra \& Debra Lilley (2015): The considerations and limitations of feedback as a strategy for behaviour change, International J ournal of Sustainable Engineering, DOI: 10.1080/ 19397038.2015.1006299

To link to this article: http:// dx. doi.org/ 10.1080/ 19397038.2015.1006299

\section{PLEASE SCROLL DOWN FOR ARTICLE}

Taylor \& Francis makes every effort to ensure the accuracy of all the information (the "Content") contained in the publications on our platform. Taylor \& Francis, our agents, and our licensors make no representations or warranties whatsoever as to the accuracy, completeness, or suitability for any purpose of the Content. Versions of published Taylor \& Francis and Routledge Open articles and Taylor \& Francis and Routledge Open Select articles posted to institutional or subject repositories or any other third-party website are without warranty from Taylor \& Francis of any kind, either expressed or implied, including, but not limited to, warranties of merchantability, fitness for a particular purpose, or non-infringement. Any opinions and views expressed in this article are the opinions and views of the authors, and are not the views of or endorsed by Taylor \& Francis. The accuracy of the Content should not be relied upon and should be independently verified with primary sources of information. Taylor \& Francis shall not be liable for any losses, actions, claims, proceedings, demands, costs, expenses, damages, and other liabilities whatsoever or howsoever caused arising directly or indirectly in connection with, in relation to or arising out of the use of the Content.

This article may be used for research, teaching, and private study purposes. Terms \& Conditions of access and use can be found at http://www.tandfonline.com/page/terms-and-conditions

It is essential that you check the license status of any given Open and Open Select article to confirm conditions of access and use. 


\title{
The considerations and limitations of feedback as a strategy for behaviour change
}

\author{
Garrath T. Wilson*, Tracy Bhamra ${ }^{1}$ and Debra Lilley ${ }^{2}$ \\ Loughborough Design School, Loughborough, UK \\ (Received 22 December 2013; accepted 9 December 2014)
}

\begin{abstract}
Design for Sustainable Behaviour (DfSB) is a maturing research area concerned with the application of design strategies to influence consumer behaviour during a products use phase towards more sustainable action. However, current DfSB research has focussed on strategy selection with little research into understanding the real-world impact of the behaviour changing interventions debated. This article presents the results of an extensive literature review of one specific DfSB strategy, feedback - a user agentive performance indicator. These findings exemplify the considerations and limitations of this particular approach to behaviour change, drawing on empirical research conducted by a breadth of authors, including two of the only medium-term case studies in the field of DfSB. Considerations discussed include the frequency, duration and accuracy of feedback; the selection of metrics and the presentation medium and mode; the use of ambience and the location of the installation. Limitations of feedback include the need for additional information and comparisons; the issue with multiple users; technical issues; relegation to background technology and the potential rebound effects. This article provides insights to both improve the effectiveness of future feedback design efforts and also to help facilitate discussion on feedbacks position as a strategy within DfSB.
\end{abstract}

Keywords: design; feedback; sustainability; domestic; energy; behaviour

\section{Introduction}

In 2008, the Parliament of the United Kingdom, as part of the UK's contribution to the collective global action required to tackle climate change, passed the Climate Change Act, including within it the target of reducing UK emissions to at least $80 \%$ of 1990 levels by 2050 (2008, 2009). Whether one considers climate change part of our moral responsibility to maintain the ecological, social and economic base for future generations or not (Bhamra and Lofthouse 2007), the reality is that the UK is still some distance from reaching its legally binding future targets on its current trajectory (Committee on Climate Change 2014).

In part, domestic energy consumption and space heating within the residential sector has contributed towards the predicament necessitating such targets (Department of Energy and Climate Change 2008, 2009). Whilst one approach would be to design more efficient technologies, prior research has shown that there is no 'silver bullet' solution, primarily due to the economic requirements of upgrading or replacing older stock with new (Darby 2006; Mintel 2009). Furthermore, this incorrectly assumes that inhabitants are passive recipients with no say or control over their environmental conditions (Chappells and Shove 2004; Cole et al. 2008). Indeed, research suggests that it is the actions and behaviours of the user that should be the target for intervention, with a focus on how a user defines comfort and enacts behaviours in its pursuit within the home (Chappells and Shove 2005; Cole et al. 2008; Shove et al. 2008; Steg and Vlek 2009).

The Carbon, Control and Comfort (CCC) project, funded by E.ON and the EPSRC Energy Efficiency panel, was a three-year, interdisciplinary UK project that attempted to reduce domestic energy comfort in social housing through the user-centred design of feedback interventions to change behaviour (EPSRC 2010). This article presents the findings from one aspect of Loughborough University's contribution to this project; a qualitative investigation into the considerations and limitations of feedback as a strategy for behaviour change, framed through the theoretical lens of Design for Sustainable Behaviour (DfSB).

\section{Design for Sustainable Behaviour}

DfSB is a branch of sustainable design theory concerned with the application of design strategies that attempt to influence consumer behaviour, during the use phase of a product, towards more sustainable action (Lilley 2009). DfSB strategies when applied to the interface between a user and their goal - the product, can be used by the designer to shape an individual's perception, learning and interaction (Tang and Bhamra 2009). This affords the opportunity to the designer to challenge the individual's intentions, facilitating conditions and habit formation, influencing the individual's actions.

\footnotetext{
*Corresponding author. Email: g.t.wilson@lboro.ac.uk
} 
Unfortunately, there is no single design approach or strategy for changing the behaviour of an individual towards more sustainable action (Lilley, Bhamra, and Lofthouse 2006; Bhamra, Lilley, and Tang 2008), however, it is recognized that there exists an axis along which these strategies are positioned, determined by the control or power in decision-making. At one end of this axis are technologically agentive solutions such as intelligent, automatic technologies, whilst the other end of the axis represents user agentive technologies, such as feedback (Wever, van Kuijk, and Boks 2008; Lilley 2009; Elias 2011; Lidman, Renström, and Karlsson 2011; Tang and Bhamra 2011; Lockton et al. 2012; Zachrisson and Boks 2012). The approach taken by Zachrisson, Storrø, and Boks (2011) encapsulates the state of the art in this respect, exploring the psychological antecedents of behaviour in order to generate a set of principles or guidelines for strategy selection. The axis or spectrum presented by Zachrisson, Storrø, and Boks (2011) is a similar convention to others in the field, although its exact axial designation (for example, control or obtrusiveness) is determined by how the resulting strategy will impact upon the individuals behaviour. The top level approaches of informing, persuading and determining are analogous to those proposed by Lilley, Bhamra, and Lofthouse (2006) and Wever, van Kuijk, and Boks (2008), with the granulation of strategies presented within this axis (from user to product in control: information, feedback, enabling, encouraging, guiding, seducing, steering, forcing and automatic) similar to those offered by Tang and Bhamra (2011).

However, as one would expect from a field that is growing rapidly with researchers investigating various facets of this axis concurrently, whilst there is some commonality, there are also still disagreements on the terminology and classification of these strategies. In addition, whilst it has been recognized that the antecedent structure of behavioural action is an important consideration in the selection of a specific behaviour changing strategy (Tang and Bhamra 2011; Zachrisson and Boks 2012), the representation, complexity and fluidity of these underlying cognitive structures makes informed and targeted selection difficult. Whilst a design process model is emerging through consensus (Selvefors, Pedersen, and Rahe 2011; Tang and Bhamra 2011; Zachrisson, Storrø, and Boks 2011), the exact relationship between the phases is yet to become standardized.

In particular, the lack of case studies at present makes evaluation difficult, with many of the implemented design processes identified (e.g. Selvefors, Pedersen, and Rahe 2011; Tang and Bhamra 2011; Zachrisson, Storrø, and Boks 2011), focussing on the early stages of the design process model and the selection or defining of DfSB strategies. Although it is understood that interventions should be evaluated against behavioural antecedents as well as sustainable and ethical impact through longitudinal study, the practical considerations and limitations of the stratagems themselves are rarely discussed within DfSB literature. Clearly different strategies have different criteria against which to design and evaluate. Taking the three points of Lilley (2009) strategies as an example, there may be a common target, such as reducing resource consumption, but the considerations and limitations of each strategy vary drastically. Eco-feedback may seek to reduce consumption through the provision of information, which has its own framing questions between itself and the user. Behaviour steering devices may rely on affordances and constraints to encourage a reduction in consumption, and thus semantics and ergonomics may be of focus. Persuasive technologies in negating the user to enforce a change may be assessed against the technical support to install and maintain the technology and to monitor the technology's effects.

The next section of this paper focuses specifically on one DfSB strategy, feedback, to exemplify considerations and limitations of this particular approach drawing on empirical research conducted by the authors and others in the field. Considerations related to evaluation, identified as a significant gap in prior art, are prioritized.

\section{Feedback}

Feedback shifts the focus towards the positive and negative consequences of behaviour and action, rather than focussing on the physiological and physical constructs prior to behaviour. By attaching either a positive or a negative consequence to behaviour, the behaviour becomes a more or less attractive option within the series of mediated intention antecedents (Abrahamse et al. 2005). In essence, feedback theory suggests that by providing the individual with feedback, a performance indicator based on the results of an enacted intention or habit, the individual can make associations between the behaviour they enact and its consequences (Abrahamse et al. 2005). Through a process of cognitive evaluation, future intentions, habits and behaviours may be influenced (Abrahamse et al. 2005; Burgess and Nye 2008).

Feedback has consistently been employed as a behavioural change intervention towards achieving a reduction in energy consumption in two ways. As a tool to illustrate the actual cost (such as time or money) of consumption and generate reflection on intention and attitude, feedback can be used to tangibly present and frame the problems caused through behavioural action. A suitably framed problem, presented through the feedback's form and delivery content may therefore influence the intention process (Fischer 2008). Information is taken in, is acted upon, and an interpretation is made (Darby 2006). Alternatively, appliance-specific feedback can be used to link a specific interaction with a product or system to energy consumption, thereby increasing an 
individual's product/system understanding and increasing individual's consciousness of their own behaviour (Fischer 2008; Darby 2010). By allowing individuals the ability to explore their own energy use and its effects, the concern/ action gap can be bridged, promoting efficiency as opposed to trying to generate an intangible sense of social obligation (Darby 2008, 2010). The key behaviour change mechanism of importance is that of information provision, as information is central to the concept of feedback as an educational tool.

What is clear is that the ability of information to motivate the individual is not only dependent on its content, but also its delivery method, as this helps to frame the information presented to the individual. It is imperative, therefore, that feedback is tailored to the intentions, capabilities and expectations of the individual, as failure to do so may lead to potentially damaging rebound effects. The process by which these mechanisms are designed needs to account for the considerations and limitation of feedback design.

\section{Feedback considerations}

Feedback can influence the energy-consuming behaviour of an individual through the provision of information, but as Wood and Newborough (2007) point out, information alone is not enough to promote action, rather it is the way in which this information is conveyed and how that motivates the individual to act. As stated, this section draws upon the work of the authors (one of only a handful of DfSB studies that have been conducted over the medium-term with users, as outlined later) and several others in the field [e.g. Fischer (2008), Abrahamse et al. (2005), Darby (2006), EDRP trials (AECOM 2011; Ofgem 2011) and Van Dam, Bakker, and Van Hal (2012)] in order to produce a comprehensive outline of the considerations for successful feedback design.

The case study of Wilson, Lilley, and Bhamra (2013) took a user-centred approach to the study of feedback devices, going through a full design process (understand and specify the context and user; intervention opportunities, intervention design, intervention evaluation) in order to understand the considerations and limitations of feedback, and to explore the ramifications for DfSB theory and practice. Based on in-depth qualitative research in seven social housing tenements in the UK, an ambient feedback device was designed and prototyped (Table 1) that had the objective of changing a targeted behaviour, the opening of windows with the heating system active, to realize a more sustainable consumption of domestic energy by the user within the defined context. This was to be achieved through a user-agentive reduction in the opening of windows with the heating system active, motivated by the users' association of the targeted behaviour with its consequences.
Table 1. Feedback intervention statuses.

\begin{tabular}{|c|c|c|c|}
\hline Information & $\begin{array}{l}\text { Window } \\
\text { status }\end{array}$ & $\begin{array}{l}\text { Radiator } \\
\text { status }\end{array}$ & $\begin{array}{l}\text { Intervention } \\
\text { light status }\end{array}$ \\
\hline The radiator is cold & Closed & $<25^{\circ} \mathrm{C}$ & Not active \\
\hline The radiator is warm & Closed & $25-43^{\circ} \mathrm{C}$ & White $^{\mathrm{a}}$ \\
\hline $\begin{array}{l}\text { The radiator is hot } \\
\text { (burn hazard) }\end{array}$ & Closed & $43^{\circ} \mathrm{C}>$ & Orange $^{\mathrm{a}}$ \\
\hline The radiator is cold & Open & $<25^{\circ} \mathrm{C}$ & Not active \\
\hline Energy conflict (waste) & Open & $25-43^{\circ} \mathrm{C}$ & $\operatorname{Red}^{\mathrm{a}}$ \\
\hline Energy conflict (waste) & Open & $43^{\circ} \mathrm{C}>$ & $\operatorname{Red}^{\mathrm{a}}$ \\
\hline
\end{tabular}

${ }^{a}$ An audible click denotes a change between statuses.

The prototype was evaluated with a 4-month user trial in two of the homes that formed the original cohort studied, in addition to 2 focus groups with a total of 10 participants.

\subsection{Frequency, duration and accuracy}

Ideally, the latest update of information should be present when the individual performs an energy-consuming act and may be open to a change in behaviour, and second when the individual chooses to acknowledge the feedback. Furthermore, research shows that the rapid provision of feedback after an action also helps to improve the cognitive linking between action/effect, thereby reinforcing the consequences of the action, and lowering consumption (Abrahamse et al. 2005; Darby 2006; Fischer 2008). Hargreaves (2010) and Fitzpatrick and Smith (2009) have shown several ways in which consumer interaction is motivated by frequency of information displayed. They report behaviour such as using the device 'hot' (using the constant feedback provided to go around the home switching devices on and off in exploration). However, as Wood and Newborough (2007) point out, not all energy-consuming activities may require the same level of frequency, with an activity such as cooking requiring a higher frequency of updates than, for example, using a washing machine.

The duration displayed by the feedback device is also an important consideration. Wood and Newborough (2007) suggest that on a display local to an action, the information should be succinct to capture immediate interest; a centralized display would show a larger time span, such as consumption over a week. As Van Dam, Bakker, and Van Hal (2012) found, the aggregation of feedback (e.g. household level/product level or real-time/ quarterly overview) can also vary dramatically based on the type of user and what they want to get out of the feedback information over time [although feedback should always be accurate, as estimated feedback disassociates the individual with the consequences of their behaviour (Hargreaves 2010)]. 
Wilson, Lilley, and Bhamra (2013) provide a clear example of this 'hot' use effect. They observed that a light indicating when the heating system was active (a hot radiator) that changed with the opening of a window local to that heat source illustrated the dynamic nature of heat loss to a participant, leading to a re-evaluation of action to conserve energy. Furthermore, the change of light in direct correlation to the surface temperature of the radiator helped a participant to understand how the heating system worked as a physical mechanism (cycling of surface temperature to maintain a consistent space temperature). This led the participant to manually explore the heating system controls throughout the home in an attempt to optimize their setting towards energy saving and comfort; the frequency and duration of update (instantaneous and parallel to event) helped to improve the link between action/effect.

\subsection{Metrics}

Energy consumption feedback can be presented to the individual through different metrics, such as energy units, cost and environmental impact. Each uses a different language to frame the context of energy consumption, thereby activating different norms and intentions within the individual (Fischer 2008).

Energy units, such as $\mathrm{kWh}$, are a standard measure for energy consumption and are generally perceived to be too abstract or difficult to relate to everyday actions (Burgess and Nye 2008; Anderson and White 2009; Hargreaves 2010; Van Dam, Bakker, and Van Hal 2012). Precise understanding, however, may not be necessary, rather it is the real-time relative movement of the energy displayed that helps a consumer 'learn what is normal, and what is not' (Anderson and White 2009; Fitzpatrick and Smith $2009,43)$. In addition, a scientific unit may instil a sense of trust (Wood and Newborough 2007). However, research suggests that cost may be a more relevant and understandable metric (Burgess and Nye 2008; Hargreaves 2010; Ofgem 2010; AECOM 2011), although those on a low-income may find such emphasis stressful (Hargreaves 2010) or even irrelevant if the outcome from expenditure, such as heating the family home, is perceived to be a basic human right (Wilson, Lilley, and Bhamra 2013). Cost may not motivate a reduction in consumption if the perceived cost of energy is considered trivial or worthless (Wood and Newborough 2007; Fitzpatrick and Smith 2009; Hargreaves 2010).

Environmental impact, for example carbon units, may be used to promote the link between action and environmental consequence. Issues with this type of metric are that the average individual does not know how to interpret the unit in comparison to their own energy consumption (Anderson and White 2009; Fitzpatrick and Smith 2009), and the unit itself is based on estimation
(Wood and Newborough 2007). In addition, individuals have been shown, in one case in particular, to be very sceptical over the merits of environmental metrics, not accepting as true the negative effect of energy consumption on the environment, believing 'all this green stuff' to be a marketing ploy, 'adding money onto your holidays' (Wilson 2013, 207).

It appears that there is no single metric that satisfies every user in terms of both understanding and intention, and that the selection of metrics should, therefore, be tailored to the intentions and capabilities of the target individual (Fischer 2008). As Wilson, Lilley, and Bhamra (2013) illustrated in their case study, the selection of temperature as a metric worked well due to the feedback interventions location and frequency of update. In short, the metric was understandable and tailored to the context.

\subsection{Presentation medium}

The medium by which information is presented also has an effect on its ability to engage with the individual, and thus be comprehended, reflected upon and effectual (Fischer 2008). Electronic media used for feedback provides flexibility of control and display, and rapid processing capabilities allowing for the presentation of real-time data. Complex devices may, conversely, be difficult for those of with a low level of education, technical ability or free time to understand or engage with (Fischer 2008). Anderson and White (2009) found that certain individuals are uncomfortable with devices that require interaction, fearing an exploration of options beyond the default display. Furthermore, the EDRP trials found that $32 \%$ of energy monitor users had difficulties in changing the default settings (AECOM 2011). Written materials, by contrast, require a lower level of education or technical ability to engage with (Fischer 2008). Feedback information accompanying a bill can also be expected to receive more careful consideration (Fischer 2008). Despite the visual quality of modern electronic displays, paper had been shown to be a preferred reading medium due to its haptic quality and freedom in how and where it is read (Holzinger et al. 2011).

As noted in the studies of both Van Dam, Bakker, and Van Hal (2012) and Wilson, Lilley, and Bhamra (2013), participants already use a type of sensory feedback to understand changes in their heating systems, using the 'click' sound of the thermostat to indicate a change in activation status. Electronic media provides the opportunity for multi-sensory feedback, including visual, auditory, tactile or olfactory feedback (e.g. smell of heated dust on a radiator). Wilson, Lilley, and Bhamra (2013) found that by replicating this 'click' sound within the feedback device to indicate a change in radiator temperature, the pre-existing capabilities and intentions of the participants could be tapped into; proving to be very 
effective in gaining the users' attention when indicating a change in state.

In brief, the medium by which information is presented should be framed within the intentions and capabilities of the individual targeted.

\subsection{Presentation mode}

In order to engage the user with the information generated, careful consideration must be given to the way in which this information is visually presented, with comprehensibility and clarity of presentation remaining clear and unambiguous.

Wood and Newborough (2007) suggest that frequency and location may affect the selected visual presentation, with numerical data better suited to frequent updates on local displays, with less frequent updates on central displays better suited to graphical data, although it should be noted that numerical displays and graphs are not always understandable by all individuals (Van Dam, Bakker, and Van Hal 2012). In addition, children may find graphical data easier to understand, potentially increasing pester power (Hargreaves 2010). Hargreaves found that the more complex the information offered, the higher the demand for active involvement, which may negate any immediate motivation to engage, a finding supported by Fitzpatrick and Smith (2009), that the preferred local display device in their trials allowed for at-a-glance information.

Research also shows a form of speedometer or traffic light system to be useful. Should the display go into the red, investigation may be prompted (Hargreaves 2010; Ofgem 2010). A focus group run by Anderson and White (2009) found that these displays show the scale, direction of change and relative position simply, emphasizing that it is the movement that grabs your attention. The work of Wilson, Lilley, and Bhamra (2013) reinforces this position, that it is the explicit, sequential change from one state to another that prompts exploration. Although they found the participants of their study to misunderstand the meaning of all of the lights, it was the change between states that primarily prompted investigation.

Whilst suitable for the specific bounded case study of Wilson, Lilley, and Bhamra (2013), Van Dam (2013, 30) suggests for a wider audience that a 'layered design in the interface' would be more appropriate, providing both at a glance further detailed information for investigation by the user, combining different modes of presentation and the ability to make comparisons (discussed later). Again, it is the intentions and capabilities of the targeted individual that are vital to be considered - as Van Dam states, 'one size does not fit all' (Van Dam 2013).

\subsection{Ambience}

Maan et al. (2011) found that light feedback realized greater energy savings than numerical feedback and that additional and unrelated cognitive load affected the time it took to process and evaluate numerical information, but not light information. This supports the theory of ambience as a provider of easy to process feedback for implicit evaluation.

Studies suggest, however, that the use of ambience alone to convey energy consumption is perceived as being ambiguous unless the ambience feature has distinguishable characteristics that can be easily cognitively mapped (Fitzpatrick and Smith 2009). Furthermore, ambience may also be construed as energy wasting or may also contradict values (Backlund et al. 2006). Investigating the effects of the same light-emitting device, Löfström and Palm found that ambient feedback is a provider of information and cues 'at a glance, from a distance' $(2008,938)$ a finding later echoed in the work of Wilson, Lilley, and Bhamra (2013), where information could be rapidly processed and understood from a distance (i.e. from across the room). At a glance, energy could be seen to be 'flying out the window' as they 'could see the colour changes straightaway', as one participant described it $(2013,20)$, thereby illustrating the impact on the room temperature without the need for lengthy interpretation. The action of a window being opened and the consequences upon the heating system was clearly understood, in a busy environment that offered many distractions (e.g. television, other family members).

\subsection{Location}

If an action requires instantaneous feedback in order to improve cognitive connections between action/effect, the device must be located to provide this. The location of the feedback device, according to Fitzpatrick and Smith (2009), Anderson and White (2009) and Ofgem's EDRP (AECOM 2011), should be installed in the individual's preferred location, which they found to be the kitchen, living room or main hallway, as this will facilitate deliberation. Van Dam, Bakker, and Van Hal suggest that the location should also account for the daily routines of the users, anecdotally described 'the baseline check' whereby participants checked their home energy management systems before going to bed to ensure that all devices were off (2012, 92-93).

A central location, however, presents a problem. If an instantaneous feedback device was positioned in an area in which the information was not immediately present to the individual, one may assume that the benefits of instantaneous delivery and interpretation of feedback would be negated. Wilson, Lilley, and Bhamra (2013) have shown that a local and visible location of an intervention not only helped to strengthen the connection between action and effect, but was also welcomed by the participants as it afforded consideration whilst being in a position of comfort. A highlighted limitation of this study 
was that the instantaneous feedback device was restricted to providing information only to the room in which it was located, which when feeding back upon a system, such as domestic heating, points towards the need for several dispersed feedback devices. However, the net impact of the reduction in energy compared to the energy cost created by the manufacture of feedback devices should be considered (Van Dam, Bakker, and Van Hal 2012).

\section{Feedback limitations}

Even if a feedback device has been designed in accordance with the above-mentioned considerations, feedback as a behaviour change strategy has its own inherent limitations that may not always be negated through design alone. This section of the article outlines these limitations.

\subsection{Additional information and comparisons}

Information through feedback works as both a supplement by which to frame consumption in addition to enhancing the conscious connection between action and its effects. Feedback itself therefore must be considered within this educational system as a means of displaying consumption, and not necessarily a means to provide the motivation level required to do so (Darby 2006; Fischer 2008). This is illustrated in the study by Wilson $(2013,203,221)$, where participants believed that certain actions are unchangeable, such as heating the home when cold or opening a window for fresh air, regardless of any information that feedback may provide.

In order to enhance the potential of feedback, additional information and further instruments may be required. Both Darby (2006) and the EDRP trials (AECOM 2011) illustrate that by combining meter or monitor readings with supplementary information on energy use, a greater reduction in consumption can occur compared with feedback alone. Additional information provides the 'how to conserve' aspect that feedback lacks (Fischer 2008). Goal setting as a mechanism can generate concentration towards an activity; physically and cognitively motivate the individual; prolong the effort required to attain the goal; and increase knowledge retrieval or creation in order to achieve the goal (McCalley, De Vries, and Midden 2011). An unrealistic goal may disenfranchise the individual from motivation, with a goal set too low limiting its effectiveness (Wood and Newborough 2007). The role of feedback in this context is to benchmark progress against goal attainment, with studies showing that by providing a goal along with relevant feedback as a point of reference, more energy can be saved than through the provision of feedback alone (McCalley 2006; McCalley, De Vries, and Midden 2011).

Furthermore, by providing a historic (a comparison of current against previous consumption) or normative (a comparison against factors that may instil normative motivations, such as other households, activities and appliances) comparison to the individual's own consumption, a context is provided by which to assess, evaluate and compete, although this could lead to negative rebound effects (Abrahamse et al. 2005; Wood and Newborough 2007; Fischer 2008).

\subsection{Multiple users}

Multiple users engaging with a singular intervention is often over looked in behaviour change studies, usually focussing on isolated participants and not including the opinions and impact upon the social nexus and associated context that surround them (e.g. their family). This may be due to gender bias towards males for more 'technical' interventions (Hargreaves 2010; Van Dam, Bakker, and Van Hal 2012) and/or that behaviour changing case studies usually focus on energy savings, driven by a single member within a household (Van Dam, Bakker, and Van Hal 2012; Wilson, Lilley, and Bhamra 2013).

Consequently, feedback interventions are not usually designed with multiple users in mind. Wilson, Lilley, and Bhamra (2013) showed that members of the same household generally had competing comfort expectations, often leading to conflict resulting in frequent ad hoc use and changing of the heating system without informing other tenants. Without any means of notification to other tenants, it would not be until the physical sensation of detecting the change in air temperature was noticed that any corrective changes could be made to lower the temperature. Although Wilson, Lilley, and Bhamra's feedback intervention allowed the primary participant (the tenant principally in control of the heating systems) the ability to detect the change in the radiator's surface temperature and optimize the system, thereby minimizing waste, the feedback was never presented to the second user - excluding them from the feedback information and decision-making process. In this respect, the feedback never accounted for multiple users nor did it tailor to their requirements, a clear limitation of this and similar feedback interventions.

\subsection{Technical issues}

If there is a failure during technical installation of a feedback device, or with the provision of accurate information, interest in the feedback or the perception of it may be reduced or damaged (Crosbie and Baker 2010; Hargreaves 2010; Van Dam, Bakker, and Van Hal 2012). Van Dam, Bakker, and Van Hal (2012) also found that when participants began to lose interest in the intervention, minor technical issues or the requirement for the participant to be proactive (e.g. changing batteries), could lead to a full rejection of the intervention. 
Furthermore, in some cases the design of the technology or the fabric of the operating environment (e.g. the home) may hinder the wireless transmission of data or simply may prevent the installation of the intervention (e.g. installing a clip on meter in a flat) (Ofgem 2010; Van Dam 2013), clearly limiting effectiveness. The intervention context needs to be understood and accounted for in the design of a behaviour changing intervention.

\subsection{Background technology}

Explored by Van Dam, Bakker, and Van Hal (2010) is the concept of background relations, and how a feedback device whose objective it is to relate the energy profile of these invisible, background technologies may in effect become one itself. This was attributed to a relapse into previous user behaviours, the increase in new energyconsuming technologies and the rebound effect.

Wilson, Lilley, and Bhamra (2013) corroborates this idea of technology relegation, showing that towards the end of their user trials that one participant used the feedback intervention less for exploration as the initial desire and period for experimentation and optimization was over. The audio-visual feedback itself became more familiar to the participant, with the once invasive feedback becoming part of daily fabric and routine, suggesting that an 'adaptive interface', or variations in feedback time of delivery and mode (Arroyo, Bonanni, and Selker 2005), may be required to stimulate ongoing interest.

Van Dam, Bakker, and Van Hal (2010) point to another direction, suggesting that feedback moving to the background may be seen as an opportunity towards automated energy management. The Design Behaviour Intervention Model (Tang and Bhamra 2011) posits that the stages of habit formation dictate how receptive an individual is to information. This suggests that feedback is most effective when the stage of habitual formation affords the intake of new information, when the individual is aware of their actions during the early stages of habit formation. As action becomes more habitual, and the user less receptive to the feedback (the intervention thus becoming a background technology), the intervention could transform along the DfSB strategy spectrum into an automated system.

\subsection{The rebound effect}

The provision of feedback and other forms of information does not always lead to a reduction in energy, as both Fischer (2008) and Abrahamse et al. (2005) discuss; if an individual is made aware of how cheap energy is or that they use a lower amount by comparison to others, they may actually increase their energy consumption. Sorrell (2007) classify the rebound effects; see Table 2.
Table 2. Classification of rebound effects (Sorrell 2007).

Classification of

rebound Summary description

Direct rebound

Increase in consumption because of the cost required to provide the efficiency measure

Substitution effect The level of on-going consumption is maintained despite switching to cheaper products or services

Income effect Increased income through efficiency savings is spent on the same product or service, increasing on-going consumption

Indirect rebound

Increase in consumption because of implementing an efficiency measure

Embodied energy The energy required to produce and

Secondary effects install the efficiency measure

Savings from the purchase of the efficiency measure may be used to purchase other consuming products and services

As a form of indirect rebound, Wilson, Lilley, and Bhamra (2013) describe how their feedback intervention highlighted to a participant when their home had run out of prepaid gas, resulting in the participant immediately topping up their prepaid supply. As an indirect consequence of the intervention, the householders had topped up their gas supply before they would have been made aware without the intervention, thereby speeding up consumption (although it could be argued that the process of evaluation may have had a longer term effect).

Regardless of the often unpredictable nature of user behaviour and the multi-stability of technology (Albrechtslund 2007), from an ethical perspective, rebound effects should always be attempted to be anticipated by the designer, as the designer of an intervention holds part responsibility for how that intervention is appropriated and used (Lilley and Wilson 2013).

\section{Discussion and conclusions}

There is an often overlooked consideration of behaviour change interventions, their effectiveness where multiple users are concerned. As highlighted by Wilson, Lilley, and Bhamra (2013), the actions of an individual can often be either conflicting or simply not in recognition of the needs of other users; in terms of feedback, the second user does not have either the opportunity to assess their own impact (due to location), or the information provided is not relevant to their intentions (tailored to the original enactor).

If an intervention attempted to deal with multiple users, the number of users and actions would increase, 
having a manifold effect on the number of variables that an intervention would need to consider to be tailored and relevant to all. One possible direction that this could take would be to tailor the interaction or information through context- and user-aware technology. Durrell Bishop in exploring the use of items tagged with RFIDs considers the connection between the user, control and the physicality of devices (Moggridge 2007); suggesting an interesting direction for bespoke interactions. A feedback intervention in this way may conceivably respond to the interactions of differing individuals with information or product configurations tailored upon their specific intentions and actions. Feedback presented in this way would help in facilitating what Van Dam, Bakker, and Van Hal refer to as a 'positive dialogue' amongst family members (2012, 95). As an issue within DfSB, the implication of multiple users should be considered further for other intervention strategies.

There is also a common assumption that certain cognitive understandings have already been formed by the individual and that this is the area of play for many interventions, especially towards the user agentive end of the DfSB spectrum with, as Zachrisson and Boks (2012) position, the informing and persuading strategies. Peeling back the layers, however, how such cognitive mapping develops needs to be considered more fully.

Taking ambient feedback as an example, literature clearly indicates that ambient feedback must be easy to cognitively map and support implicit evaluation. Are we to assume that an individual has a clear mental model of how the feedback relates to their action and consequence prior to initial interaction with the feedback device, or should we provide a mechanism through which this cognitive relationship can be developed, and perhaps more appropriately, be shaped? Interestingly, Wilson, Lilley, and Bhamra (2013) showed how participants had generated their own cognitive maps in parallel to receiving feedback on action; relating the feedback offered to physical sensation. Initially it was found that ambient feedback was only a prompt for the participants to touch the radiator to determine its temperature, however, over the course of the user trials the participants began to accept that certain touch temperatures related to specific lights and sounds, generating the desired implicit evaluation; eventually the lights replaced touching the radiator all together. The combination of feedback and physical stimulus had created a new cognitive mapping between temperature and light that previously had not existed, suggesting an interesting direction for the shaping of an individual's perception and interaction with information.

In some respects, this period of finding and generating of understanding by the individual draws parallels with the work of Routarinne and Redström (2007), who apply the concept of domestication to understand how the individual creates new meaning in intervention technologies through reference to their intentions and context. Applying this concept to feedback, it would appear that feedback information does not have a static meaning, but is shaped by the individual over time thus affecting the perception and framing of the problem and resulting action. The concept of feedback as a dynamic mechanism for behaviour change is also suggested within the work of Zachrisson, Storr $\varnothing$, and Boks (2011) and Tromp, Hekkert, and Verbeek (2011), who contend that the distribution of control spectrum is not a static axis at all but changes over time as the individual's perception of the intervention changes. Feedback's position, therefore, may be fluid.

Also, an interesting point to consider is whether a form of ambient feedback that has developed a strong associated habitual response is still in fact within the users' control and decision-making capabilities and is not, at its most extreme, a form of conditioned user automation? One could easily envisage an axis whereby the two extreme poles (user and product) are automated, one through cognitive mechanisms and the other through mechanical mechanisms. Each strategy on this DfSB axis could potentially have a sliding scale of effectiveness and control dependent on cognitive process over time.

Finally, in feedback studies, the $5-15 \%$ reduction with direct feedback as cited by Darby (2006) has commonly become the golden target. However, although focussing on per cent reduction targets does appear to carry some logic (as this is how the Climate Change Act 2008 (2009) is framed, for example) focussing on per cent savings as a meter of the success of an intervention is an ill-advised tactic. This precludes any debate over the actual success of the mechanism itself for behaviour change and inhibits progress towards better understanding and design.

Within feedback studies specifically, the design process and the actual behavioural impact of the intervention itself need to be more fully considered and reported. Did the intervention function as intended? Has the user's behaviour actually changed? Is that change actually sustainable and ethical? It is important to remember, a change in behaviour does not necessarily correlate to a change in energy consumption, especially when one considers rebound effects. As explored elsewhere (Wilson, Lilley, and Bhamra 2013; Lilley and Wilson 2013), and especially given the context of this special issue, there must at least be a suitable evaluation of the design, sustainable and behaviour aspects that comprise DfSB theory.

\section{Acknowledgements}

The authors thank the Engineering and Physical Sciences Research Council (EPSRC) and E.ON UK for providing the financial support for this study as part of the Carbon, Control \& Comfort project (EP/G000395/1). 


\section{Disclosure statement}

No potential conflict of interest was reported by the authors.

\section{Notes}

1. Email: T.Bhamra@lboro.ac.uk

2. Email: D.Lilley@lboro.ac.uk

\section{References}

Abrahamse, Wokje, Linda Steg, Charles Vlek, and Talib Rothengatter. 2005. "A Review of Intervention Studies Aimed at Household Energy Conservation." Journal of Environmental Psychology 25 (3): 273-291. doi: 10.1016/j. jenvp.2005.08.002.

AECOM. 2011. "Energy Demand Research Project: Final Analysis." Ofgem.

Albrechtslund, A. 2007. "Ethics and Technology Design." Ethics and Information Technology 9 (1): 63-72. doi:10.1007/ s10676-006-9129-8.

Anderson, W., and V. White. 2009. Exploring Consumer Preferences for Home Energy Display Functionality: Report to the Energy Saving Trust. Bristol: Centre for Sustainable Energy.

Arroyo, E., L. Bonanni, and T. Selker. 2005. "Waterbot: Exploring Feedback and Persuasive Techniques at the Sink." CHI 2005, Portland, Oregon, USA, April 2-7, 2005.

Backlund, S., M. Gyllenswärd, A. Gustafsson, S. Ilstedt Hjelm, R. Mazé, and J. Redström. 2006. "STATIC! The Aesthetics of Energy in Everyday Things." WonderGround - Design Research Society International Conference, Lisbon, Portugal.

Bhamra, T. A., D. Lilley, and T. Tang. 2008. "Sustainable Use: Changing Consumer Behaviour Through Product Design." Changing the Change, Turin, Italy, July 11-12, 2008.

Bhamra, T. A., and V. A. Lofthouse. 2007. "Design for Sustainability a Practical Approach." In Design for Social Responsibility, edited by Rachel Cooper. Burlington, VT: Gower.

Burgess, Jacquelin, and Michael Nye. 2008. "Re-Materialising Energy Use Through Transparent Monitoring Systems.” Energy Policy 36 (12): 4454-4459. doi: 10.1016/j.enpol.2008.09.039.

Chappells, Heather, and Elizabeth Shove. 2004. "Comfort: A Review of Philosophies and Paradigms." The University of Lancaster.

Chappells, Heather, and Elizabeth Shove. 2005. "Debating the Future of Comfort: Environmental Sustainability, Energy Consumption and the Indoor Environment." Building Research \& Information 33 (1): 32-40. doi: 10.1080/ 0961321042000322762.

Cole, Raymond J., John Robinson, Zosia Brown, and Meg O'Shea. 2008. "Re-Contextualizing the Notion of Comfort." Building Research \& Information 36 (4): 323-336. doi: 10.1080/09613210802076328.

Committee on Climate Change. 2014. "Meeting Carbon Budgets: 2014 Progress Report to Parliament.".

Crosbie, Tracey, and Keith Baker. 2010. "Energy-Efficiency Interventions in Housing: Learning from the Inhabitants." Building Research \& Information 38 (1): 70-79. doi: 10. 1080/09613210903279326.

Darby, Sarah. 2006. "The Effectiveness of Feedback on Energy Consumption." In A Review for DEFRA of the Literature on Metering, Billing and Direct Displays. Oxford: Environmental Change Institute, University of Oxford.
Darby, Sarah. 2008. "Why, What, When, How, Where and Who? Developing UK Policy on Metering, Billing and Energy Display Devices." ACEEE Summer Study on Energy Efficiency in Buildings, Asilomar, CA, August $17-22$.

Darby, Sarah. 2010. Literature Review for the Energy Demand Research Project. London: Ofgem (Office of Gas and Electricity Markets.

Department of Energy and Climate Change. 2008. "2008 Greenhouse Gas Emissions, Final Figures by End-User." Department of Energy and Climate Change.

Department of Energy and Climate Change. 2009. "Energy Metering: A Consultation on Smart Metering for Electricity and Gas." Department of Energy and Climate Change.

Elias, E. W. A. 2011. "User-Efficient Design: Reducing the Environmental Impact of User Behaviour Through the Design of Products." Doctor of Philosophy, Department of Mechanical Engineering, University of Bath.

EPSRC. 2010. "Carbon, Control and Comfort: User-Centred Control Systems for Comfort, Carbon Saving and Energy Management, EPSRC Reference: EP/G000395/1." Last Modified June 2, 2010. Accessed January 05, 2015. http:// gow.epsrc.ac.uk/NGBOViewGrant.aspx?GrantRef=EP/ G000395/1

Fischer, C. 2008. "Feedback on Household Electricity Consumption: A Tool for Saving Energy?" Energy Efficiency 1 (1): 79-104. doi: 10.1007/s12053-008-9009-7.

Fitzpatrick, G., and G. Smith. 2009. "Technology-Enabled Feedback on Domestic Energy Consumption: Articulating a Set of Design Concerns." IEEE Pervasive Computing 8 (1): 37-44. doi: 10.1109/MPRV.2009.17.

Hargreaves, T. 2010. "The Visible Energy Trial: Insights from Qualitative Interviews." In Tyndall Centre Working Papers. Norwich: Tyndall Centre for Climate Change Research.

Holzinger, A., M. Baernthaler, W. Pammer, H. Katz, V. BjelicRadisic, and M. Ziefle. 2011. "Investigating Paper vs. Screen in Real-Life Hospital Workflows: Performance Contradicts Perceived Superiority of Paper in the User Experience." International Journal Human-Computer Studies 69 (9): 563-570. doi: 10.1016/j.ijhes.2011.05.002.

Lidman, K. M. E., S. E. Renström, and I. C. MA Karlsson. 2011. "The Green User: Design for Sustainable Behaviour." Diversity and Unity, IASDR2011, The 4th World Conference on Design Research, Delft, The Netherlands, October 31-November 4.

Lilley, D. 2009. "Design for Sustainable Behaviour: Strategies and Perceptions." Design Studies 30 (6): 704-720. doi: 10. 1016/j.destud.2009.05.001.

Lilley, D., T. A. Bhamra, and V. A. Lofthouse. 2006. "Towards Sustainable Use: An Exploration of Designing for Behavioural Change." DeSForm 2006: European Workshop on Design and Semantics of Form and Movement, Eindhoven, The Netherlands.

Lilley, D., and G. T. Wilson. 2013. "Integrating Ethics into Design for Sustainable Behaviour." Journal of Design Research 11 (3): 278-299. doi: 10.1504/JDR.2013.056593.

Lockton, Dan D., David D. Harrison, Neville A. Stanton, Dan Lockton, and David Harrison. 2012. "Models of the User: Designers' Perspectives on Influencing Sustainable Behaviour." Journal of Design Research 10 (1/2): 7-27. doi: 10.1504/JDR.2012.046137.

Löfström, E., and J. Palm. 2008. "Visualising Household Energy Use in the Interest of Developing Sustainable Energy Systems." Housing Studies 23 (6): 935-940. 
Maan, S., B. Merkus, J. Ham, and Cees J. H. Midden. 2011. "Making it Not Too Obvious: The Effect of Ambient Light Feedback on Space Heating Energy Consumption." Energy Efficiency 4 (2): 175-183. doi: 10.1007/s12053010-9102-6.

McCalley, L. T. 2006. "From Motivation and Cognition Theories to Everyday Applications and Back Again: The Case of Product-Integrated Information and Feedback." Energy Policy 34 (2): 129-137. doi: 10.1016/j.enpol.2004.08.024.

McCalley, L. T., P. W. De Vries, and Cees J. H. Midden. 2011. "Consumer Response to Product-Integrated Energy Feedback: Behavior, Goal Level Shifts, and Energy Conservation." Environment and Behavior 43 (4): 525-545. doi: 10. 1177/0013916510371053.

Mintel. 2009. "Domestic Central Heating (Industrial Report) UK - May 2009.".

Moggridge, Bill. 2007. Designing Interactions. Cambridge, MA: The MIT Press.

Ofgem. 2010. "Energy Demand Research Project." Review of Progress for the Period March 2009-September 2009.

Ofgem. 2011. "Consumer Bulletin: Getting Smart: Preparing for Change.".

Parliament of the United Kingdom. 2008. "Climate Change Act 2008." Chapter 27. The Stationery Office Limited.

Parliament of the United Kingdom. 2009. "The Climate Change Act 2008 (2020 Target, Credit Limit and Definitions) Order 2009." Order No. 1258. The Stationery Office Limited.

Routarinne, S., and J. Redström. 2007. "Domestication as Design Intervention." Design Inquiries 2007, Stockholm, Sweden, May 27-30.

Selvefors, A., K. B. Pedersen, and U. Rahe. 2011. "Design for Sustainable Behaviour: Systemising the Use of Behavioural Intervention Strategies." Designing Pleasurable Products and Interfaces, Milan, Italy, June 22-25, 2011.

Shove, Elizabeth E., Heather Chappells, Loren Lutzenhiser, Bruce Hackett, and Elizabeth Shove. 2008. "Comfort in a Lower Carbon Society." Building Research \& Information 36 (4): 307-311. doi: 10.1080/09613210802079322.

Sorrell, S. 2007. "The Rebound Effect: An Assessment of the Evidence for Economy-Wide Energy Savings from Improved Energy Efficiency." UKERC.

Steg, Linda, and Charles Vlek. 2009. "Encouraging ProEnvironmental Behaviour: An Integrative Review and Research Agenda." Journal of Environmental Psychology 29 (3): 309-317. doi: 10.1016/j.jenvp.2008.10.004.

Tang, T., and T. A. Bhamra. 2009. "Understanding Consumer Behaviour to Reduce Environmental Impacts Through
Sustainable Product Design.” Undisciplined! Design Research Society Conference 2008, Sheffield, UK, July 16-19, 2008.

Tang, T., and T. A. Bhamra. 2011. "Applying a Design Behaviour Intervention Model to Design for Sustainable Behaviour." The Tao of Sustainability: An International Conference on Sustainable Design in a Globalization Context, Beijing, China, October 27-29, 2011.

Tromp, N., P. Hekkert, and P. P. Verbeek. 2011. "Design for Socially Responsible Behavior: A Classification of Influence Based on Intended User Experience.” Design Issues 27 (3): 3-19. doi: 10.1162/DESI_a_00087.

Van Dam, S. S. 2013. Smart Energy Management for Households: A Practical Guide for Designers, HEMS Developers, Energy Providers, and the Building Industry. CreateSpace Independent Publishing Platform.

Van Dam, S. S, C. Bakker, and J. D. M. Van Hal. 2010. "Home Energy Monitors: Impact Over the Medium-Term." Building Research \& Information 38 (5): 458-469. doi: 10.1080/ 09613218.2010 .494832$.

Van Dam, S. S., C. Bakker, and J. D. M. Van Hal. 2012. "Insights Into the Design, Use and Implementation of Home Energy Management Systems." Journal of Design Research 10 (1/2): 86-101. doi: 10.1504/JDR.2012.046141.

Wever, Renee, Jasper van Kuijk, and Casper Boks. 2008. "UserCentred Design for Sustainable Behaviour." International Journal of Sustainable Engineering 1 (1): 9-20. doi: 10.1080/19397030802166205.

Wilson, G. T. 2013. "Design for Sustainable Behaviour: Feedback Interventions to Reduce Domestic Energy Consumption." Doctor of Philosophy, Loughborough Design School, Loughborough University.

Wilson, G. T., D. Lilley, and T. A. Bhamra. 2013. "Design Feedback Interventions for Household Energy Consumption Reduction." Proceedings of ERSCP-EMSU 2013 Conference, Istanbul, Turkey, June 4-7, 2013.

Wood, G., and M. Newborough. 2007. "Energy-Use Information Transfer for Intelligent Homes: Enabling Energy Conservation with Central and Local Displays." Energy and Buildings 39 (4): 495-503. doi: 10.1016/j.enbuild.2006.06.009.

Zachrisson, J., and C. Boks. 2012. "Exploring Behavioural Psychology to Support Design for Sustainable Behaviour Research." Journal of Design Research 10 (1/2): 50-66. doi: 10.1504/JDR.2012.046139.

Zachrisson, J., Gøril Storrø, and C. Boks. 2011. "Using a Guide to Select Design Strategies for Behaviour Change; Theory vs. Practice." Proceedings of EcoDesign 2011 International Symposium, Kyoto, Japan. 